\title{
Sporadic and epidemic community legionellosis: two faces of the same illness
}

\author{
N. Sopena*, L. Force”, M.L. Pedro-Botet*, P. Barrufet”, G. Saucađ, M. García- \\ Núñez*, G. Tolchinsky*, J.A. Capdevila" ${ }^{\#}$ and M. Sabrià*
}

ABSTRACT: The present study compares the risk factors, presentation and outcome of community-acquired Legionella pneumophila pneumonia in 138 sporadic-case patients (19942004) and 113 outbreak-case patients (2002) treated in two hospitals in Catalonia (Spain) since urinary antigen assays were adopted.

Univariate and multivariate analysis were performed to compare epidemiological and clinical features, blood chemistry values, radiological findings and outcome of sporadic and epidemic legionnaires' disease.

Univariate analysis showed that male sex, chronic lung disease, HIV infection and immunosuppressive therapy prevailed in sporadic cases. Presentation with respiratory symptoms, confusion and blood chemistry alterations, such as hyponatraemia, aspartate aminotransferase and blood urea nitrogen elevation, and partial pressure of oxygen $\mathrm{PO}_{2}<7.98 \mathrm{KPa}$ $(60 \mathrm{mmHg})$ were also more frequent in sporadic cases, while headache prevailed in outbreak cases. Sporadic cases had a greater delay in treatment, were more severe and had a worse outcome than epidemic cases. Multivariate analysis showed significant differences in sex, chronic lung disease, HIV infection and headache.

The clinical and outcome differences between the two groups may be explained by the detection of milder forms of legionnaires' disease, the earlier treatment and the lower severity of underlying disease in the outbreak cases.

KEYWORDS: Community-acquired pneumonia, Legionella, Legionella pneumophila, legionnaires' disease, outbreak, urinary antigen

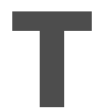
he incidence of legionnaires' disease has increased in the last decade since the introduction of urinary antigen immunoassays [1, 2]. This test accounts for most of the diagnostics due to its high sensitivity and ease of use [3]. Legionella pneumophila has become one of the leading causes of community-acquired pneumonia in adults, accounting for $6-14 \%$ of cases requiring hospitalisation in recent studies $[4,5]$. Legionnaires' disease occurs sporadically and in outbreaks, with the sporadic form representing $65-82 \%$ of the cases $[1,2,6]$. Nevertheless, the number of confirmed community outbreaks, including several with $>100$ cases, has increased in recent years due to the use of Legionella antigenuria $[2,6]$.

Routine testing for Legionella urinary antigen has increased the number of diagnostics of legionnaires' disease and has allowed earlier diagnosis and treatment, greatly improving the prognosis [7]. This has been particularly true for milder cases, mainly in the outbreak setting [8]. However, most of the knowledge on risk factors, clinical presentation and outcome of community-acquired legionnaires' disease is based in studies performed before routine urinary antigen testing was adopted $[9,10]$. Moreover, recent community outbreaks have contributed to the better understanding of legionnaires' disease in this setting [11-13].

There are no comparative studies of the characteristics of sporadic and outbreak-related legionnaires' disease. In theory, the risk factors and the clinical presentation of epidemic legionellosis may differ from those of sporadic forms due to its higher attack rate and the fact that the diagnosis of a proportion of milder cases may go undetected in a nonoutbreak setting. During an outbreak, the physicians' greater awareness of the range of clinical presentations may promote earlier diagnosis and treatment and, consequently, modify the outcome of legionellosis [8, 9]. Thus, the objective of the present study is to compare the risk factors, the clinical presentation and the outcome of community-acquired legionnaires' disease, with respect to sporadic and outbreak context in the years since the
AFFILIATIONS

*Infectious Diseases Unit, Germans Trias i Pujol University Hospital, Badalona,

\# Internal Medicine Department, and - Microbiology Laboratory, Mataró Hospital, Mataró, Barcelona, Spain.

\section{CORRESPONDENCE}

N. Sopena

Unitat de Malalties Infeccioses, Hospital Universitari Germans Trias i Pujol. C/ Canyet s/n. 08916 Badalona Barcelona Spain

Fax: 34934978843

E-mail: nsopena.germanstrias@

gencat.net

Received:

June 132006

Accepted after revision:

September 092006

SUPPORT STATEMENT

This article has been presented in part at the 15th European Congress of Clinical Microbiology and Infectious Diseases, Copenhagen (Denmark), April 5, 2005. 
present authors have been able to implement antigenuria immunoassays.

\section{PATIENTS AND METHODS Setting}

Since 1994, the present authors have prospectively studied the cases of community-acquired legionnaires' disease admitted to the Germans Trias i Pujol Hospital, a 650-bed tertiary centre located in Badalona (north Barcelona) that serves an urban area of 700,000 inhabitants with 22,000 admissions annually. The Legionella urinary antigen assay has been used in the diagnosis of community-acquired pneumonia in the authors' hospital since 1994. Some aspects of the present study have already been published elsewhere $[6,14]$.

Between July and August 23, 2002, an explosive outbreak of community-acquired legionnaires' disease developed in Mataró, a manufacturing centre and seaport north-east of Barcelona. This incident involved $\geqslant 154$ people, 113 of whom had definitive (confirmed) legionnaires' disease [15].

\section{Patients}

The present study included 138 patients with sporadically community-acquired legionnaires' disease, prospectively diagnosed from 1994 to 2004 at the Germans Trias i Pujol Hospital, and 113 patients with L. pneumophila pneumonia conclusively diagnosed within the Mataró outbreak.

A confirmed case of legionnaires' disease was defined as a case of pneumonia with laboratory evidence of acute infection with Legionella, including: isolation from respiratory samples; a four-fold or higher rise in antibody titers from 1:128 against $L$. pneumophila; a serogroup 1-6 by immunofluorescence in paired acute and convalescent phase serum specimens; or detection of L. pneumophila serogroup 1 in urine by ELISA or immunochromatographic test (ICT).

\section{Microbiological diagnosis}

Urinary antigen detection of L. pneumophila serogroup 1 by ELISA or ICT was positive in 117 of the 138 sporadic cases and in 110 of the 113 outbreak cases. L. pneumophila was isolated from the sputum of 10 sporadic cases and 10 outbreak cases. Seroconversion was detected in $43(31.1 \%)$ sporadic cases and in $24(21.2 \%)$ outbreak cases. Some cases were diagnosed according to more than one of the mentioned tests.

\section{Variables studied}

The following variables were studied: 1 ) demographic (age and sex); 2) individual risk factors (including cigarette smoking and alcohol abuse); 3) underlying diseases, such as chronic lung disease, chronic heart disease, diabetes mellitus, liver cirrhosis, neoplasm, chronic renal failure and HIV infection; 4) pharmacological immunosuppressive therapy (corticosteroids or chemotherapy); 5) clinical features, laboratory data and radiological findings on presentation; 6) Fine score risk category, need for hospital and intensive care unit admission, and delay in treatment; 7) type, duration and efficacy of antibiotic treatment; and 8) outcome (time to apyrexia, and/or complications, cure, recurrence or death related to pneumonia).

Patients in Fine score risk classes I and II, contrary to classes III-V, are defined as having sufficiently low risk of death, or other adverse medical outcomes, that the physician can

consider outpatient treatment [16]. Delay in treatment was defined as the number of days from the onset of illness (fever, if present) to administration of appropriate antibiotic therapy. Time to apyrexia was defined as the hours of fever following the initiation of appropriate antibiotic treatment. Antibiotic treatment was considered adequate when it included a quinolone or a macrolide. Death was considered to be related to pneumonia when it was directly caused by pneumonia or its complications.

\section{Statistical methods}

Univariate analysis was performed using a paired t-test when comparing quantitative variables and a Chi-squared test for qualitative variables. In all cases, significance was defined as a p-value $\leqslant 0.05$. All variables found to be significant on univariate analysis and all clinically important variables were included in the multivariate logistic regression analysis.

\section{RESULTS}

\section{Demographic data and risk factors}

Male sex, some underlying diseases (including chronic lung diseases and HIV infection) and a history of immunosuppressive therapy (mainly with corticosteroids) were significantly more frequent in sporadic cases than in outbreak cases. A history of alcohol abuse was also more frequent in sporadic cases, although this variable did not achieve statistical significance on univariate analysis. Conversely, $113(81.9 \%)$ of the sporadic cases and $92(81.4 \%)$ of the outbreak cases had known individual risk factors for legionnaires' disease, including cigarette smoking, alcohol abuse and/or underlying diseases (table 1)

\section{Clinical presentation, analytical data and radiological findings}

Clinical presentation with respiratory symptoms, such as cough, expectoration, thoracic pain, dyspnoea and confusion,

\section{TABLE 1 Demographic data and risk factors}

Variable

Subjects $n$

Age yrs

Age $>60$ yrs

Male

Individual risk factors

Cigarette smoking

Alcohol abuse

Underlying diseases

Chronic lung disease

Chronic heart disease

Diabetes

Liver cirrhosis

Neoplasm

HIV infection

Chronic renal failure

Corticoid treatment
Sporadic cases Outbreak cases p-value

$\begin{array}{ccc}138 & 113 & \\ 56.6 \pm 15.5 & 59.5 \pm 16.6 & 0.1 \\ 60(43.5) & 59(52.2) & 0.2 \\ 113(81.9) & 73(64.6) & 0.002^{\#} \\ 113(81.9) & 92(81.4) & 1 \\ 67(48.6) & 51(48.1) & 1 \\ 37(26.8) & 17(16.5) & 0.08 \\ 76(55.1) & 56(49.6) & 0.4 \\ 27(19.6) & 11(9.7) & 0.04^{\#} \\ 17(12.4) & 13(11.5) & 0.9 \\ 21(15.2) & 27(23.9) & 0.1 \\ 8(5.9) & 10(8.8) & 0.5 \\ 10(7.3) & 10(8.8) & 0.8 \\ 16(11.6) & 2(1.8) & 0.003^{\#} \\ 3(2.2) & 2(1.8) & 0.8 \\ 8(5.8) & 0 & 0.009^{\#}\end{array}$

Data are presented as mean $\pm S D$ and $n(\%)$, unless otherwise stated. Statistical significance in univariate analysis. 


\begin{tabular}{|c|c|c|c|}
\hline Variable & Sporadic cases & $\begin{array}{l}\text { Outbreak } \\
\text { cases }\end{array}$ & $p$-value \\
\hline Subjects n & 138 & 113 & \\
\hline Fever & $134(97.1)$ & $112(99.1)$ & 0.4 \\
\hline Days of fever & $4.3 \pm 2.6$ & $4.04 \pm 1.9$ & 0.4 \\
\hline Cough & 97 (70.3) & $58(51.8)$ & $0.004^{\#}$ \\
\hline Expectoration & $54(39.1)$ & $15(14)$ & $<0.001^{\#}$ \\
\hline Thoracic pain & $32(23.2)$ & $14(12.6)$ & $0.04^{\#}$ \\
\hline Dyspnoea & $62(44.9)$ & $24(28.6)$ & $0.02^{\#}$ \\
\hline Headache & $35(25.5)$ & $61(56.5)$ & $<0.001^{\#}$ \\
\hline Confusion & $23(16.7)$ & $8(7.5)$ & $0.04^{\#}$ \\
\hline Diarrhoea & $29(21)$ & $21(18.9)$ & 0.8 \\
\hline $\mathrm{WBC} \cdot \mathrm{mm}^{-3}>12000$ & $64(48.1)$ & 48 (43.6) & 0.6 \\
\hline $\mathrm{Na}<130 \mathrm{mmol} \cdot \mathrm{L}^{-1}$ & 27 (21.3) & $7(6.7)$ & $0.003^{\#}$ \\
\hline $\mathrm{CK}>232 \mathrm{U} \cdot \mathrm{L}^{-1}$ & $30(27.5)$ & $5(13.5)$ & 0.1 \\
\hline AST $>37 \mathrm{U} \cdot \mathrm{L}^{-1}$ & $62(48.8)$ & 30 (31.3) & $0.01^{\#}$ \\
\hline $\mathrm{BUN} \geqslant 13 \mathrm{mmol} \cdot \mathrm{L}^{-1}$ & $18(14.6)$ & $2(2)$ & $0.001^{\#}$ \\
\hline $\mathrm{PO}_{2}<60 \mathrm{mmHg}$ & $60(45.8)$ & $35(31)$ & $0.02^{\#}$ \\
\hline $\begin{array}{l}\geqslant 2 \text { lobes radiographic } \\
\text { extension }\end{array}$ & $26(19.1)$ & $10(9.3)$ & $0.04^{\#}$ \\
\hline
\end{tabular}

Data are presented as $n(\%)$ and mean $\pm S D$, unless otherwise stated. WBC: white blood cell; CK: creatinine kinase; AST: aspartate aminotransferase; BUN: blood urea nitrogen; $\mathrm{PO}_{2}$ : oxygen partial pressure. ${ }^{\#}$ : Statistical significance in univariate analysis. $1 \mathrm{mmHg}=0.133 \mathrm{KPa}$.

prevailed in sporadic cases. Headache was significantly more frequently observed in outbreak cases (table 2).

Blood chemistry alterations on presentation, such as hyponatraemia $\left(\mathrm{Na}<130 \mathrm{mmol} \cdot \mathrm{L}^{-1}\right)$, aspartate aminotransferase (AST) elevation, an increase in blood urea nitrogen (BUN) $\geqslant 13 \mathrm{mmol} \cdot \mathrm{L}^{-1}$ and a $\mathrm{PO}_{2}<7.98 \mathrm{KPa}(60 \mathrm{mmHg})$ were significantly more frequent in sporadic cases. Presentation with radiographic evidence of bi-lobar or multi-lobar infiltrates on chest radiographs was also significantly more frequent in sporadic cases than in outbreak cases: $19.1 \%$ versus $9.3 \%$, respectively (table 2 ).

\section{Treatment and outcome}

Placement in Fine score risk class III, IV or V was significantly more frequent in sporadic cases than in outbreak cases. Hospital admission was needed for 138 sporadic cases $(100 \%)$ compared with 68 outbreak cases (60\%). Intensive care unit (ICU) admission was required for $16.1 \%$ of the sporadic cases versus $4.4 \%$ of the cases related to the outbreak (table 3 ).

The delay before receiving adequate treatment was significantly longer in sporadic cases than in outbreak cases. Although significantly more patients were treated with macrolides than with quinolones, no significant difference was found between the percentages of sporadic and outbreak patients who received a course of appropriate treatment. However, the mean duration of treatment was longer for outbreak patients (table 3 ).

Regarding outcome, the incidence of complications such as respiratory failure, need of mechanical ventilation, acute renal
TABLE 3 Treatment and outcome

\begin{tabular}{lccc} 
Variable & Sporadic cases & Outbreak cases & p-value \\
\hline Subjects n & 138 & 113 & \\
Fine score classes III-V & $48 / 83(57.8)$ & $34 / 83(41)$ & $0.04^{\#}$ \\
Hospital admission & $138(96.4)$ & $68(60)$ & $0.003^{\#}$ \\
ICU admission & $22(16.1)$ & $5(4.4)$ & $0.004^{\#}$ \\
Delay in treatment days & $5 \pm 2.8$ & $4.2 \pm 2.4$ & $0.02^{\#}$ \\
Adequate antibiotic treatment & $124 / 127(97.6)$ & $113(100)$ & 0.2 \\
Days of treatment & $16.9 \pm 6.1$ & $14.76 \pm 2.5$ & $0.001^{\#}$ \\
Treatment with macrolides & $77 / 124(62.1)$ & $109(96.4)$ & $0.001^{\#}$ \\
$\quad$ versus quinolones & $47 / 124(37.9)$ & $4(3.5)$ & \\
Time to apyrexia h & $59.7 \pm 67.4$ & $57.8 \pm 30.8$ & 0.80 \\
Complications & $75(54.3)$ & $37(32.7)$ & $0.001^{\#}$ \\
$\quad$ Respiratory failure & $70(50.7)$ & $37(32.7)$ & $0.006^{\#}$ \\
$\quad$ Mechanical ventilation & $15(10.9)$ & $4(3.5)$ & $0.03^{\#}$ \\
Acute renal failure & $10(7.4)$ & 0 & $0.002^{\#}$ \\
$\quad$ Septic shock & $11(8)$ & $2(1.8)$ & $0.04^{\#}$ \\
Evolution & & & \\
$\quad$ Cure & $128(92.6)$ & $111(98.2)$ & \\
Death related to pneumonia & $8(5.9)$ & $2(1.8)$ & 0.1 \\
$\quad$ Recurrence & $2(1.5)$ & & \\
\hline
\end{tabular}

Data are presented as $n(\%)$ and mean \pm SD. ICU: intensive care unit. *: Statistical significance in univariate analysis.

failure and septic shock was significantly higher in sporadic cases compared with outbreak cases. Recurrences occurred in two sporadic cases. Decline to death occurred in $5.9 \%$ of sporadic cases compared with $1.8 \%$ in the outbreak cases (table 3).

On multivariate analysis only the variables sex, chronic lung disease, HIV infection and headache remained significant (table 4).

\section{DISCUSSION}

To the best of the authors' knowledge, the present study is the first study of Legionella pneumonia to compare sporadic and outbreak presentation since the advent of antigenuria testing. The results of the present study show that patients with sporadic community-acquired legionnaires' disease were more frequently male and had a higher prevalence of chronic lung disease, HIV infection and corticosteroid therapy compared

\section{TABLE 4 Multivariate analysis}

\begin{tabular}{lcl} 
Variable & p-value & OR (95\% Cl) \\
\hline Sex male $^{\#}$ & 0.05 & $2.56(1.32-4.94)$ \\
Chronic lung disease $^{\#}$ & 0.01 & $2.67(1.20-5.94)$ \\
HIV infection $^{\#}$ & 0.004 & $9.75(2.09-45.48)$ \\
Headache $^{*}$ & 0.006 & $2.79(1.34-5.80)$ \\
\hline
\end{tabular}

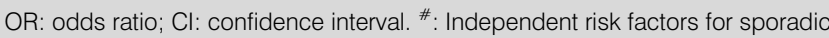
legionnaires' disease; ': independent risk factor for epidemic legionnaires' disease. 
with outbreak-associated cases. Presentation with respiratory symptoms, confusion and blood chemistry alterations, such as hyponatraemia $<130 \mathrm{mmol} \cdot \mathrm{L}^{-1}$, AST elevation, BUN increase $\geqslant 13 \mathrm{mmol} \cdot \mathrm{L}^{-1}$ and $\mathrm{PO}_{2}<7.98 \mathrm{KPa}(60 \mathrm{mmHg})$, was more frequent in sporadic cases, while headache prevailed in outbreak cases. Finally, sporadic legionnaires' disease was more severe and had a worse outcome than the epidemic disease.

Several risk factors for acquisition of legionnaires' disease have been identified in observational and case-control studies. These include: advanced age, male sex, cigarette smoking, alcohol abuse and underlying diseases, such as chronic lung disease, neoplasm, diabetes, HIV infection and immunosuppressive therapy, especially with corticosteroids $[9,10,12,13$, 17]. In a similar manner to other studies, most of the sporadic and epidemic cases in the present study had one or more of the known risk factors for legionellosis, including cigarette smoking, alcohol abuse and underlying diseases [11, 13, 1822]. This fact highlights the importance of the susceptibility of the population, even with the higher degree of exposure, to the source of Legionella that occurs in an outbreak setting. In particular, nearly half of the patients in the two groups were smokers, a fact that emphasises the increased susceptibility to legionnaires' disease in individuals with compromised mucociliary clearance in the tracheobroncheal tree $[12,17]$. However, it is noteworthy that only half of the patients in the two groups had underlying diseases and were $>60$ yrs of age. This fact is in contrast with the high prevalence of advanced age or underlying diseases observed in Streptococcus pneumoniae pneumonia, thereby emphasising the importance of general immunity in pneumococcal infection [23, 24].

The higher ratio of males in sporadic cases observed in the present study may be explained by the higher prevalence of some underlying risk factors. It has been argued that the greater prevalence of cigarette smoking and its complications in males may predispose Legionella infection by deterioration of the respiratory mucosa [17]. Conversely, the higher frequency of chronic pulmonary disease, HIV infection and immunosuppressive therapy observed in sporadic cases may at least partly account for the greater susceptibility of these patients to Legionella infection, even with exposure to a small inoculum $[18,25]$. Moreover, patients with severe underlying diseases might be expected to be referred more frequently to the hospital for diagnosis of sporadic community-acquired pneumonia.

In the present study, a more atypical presentation of legionnaires' disease was observed in the outbreak cases, with lower incidence of respiratory symptoms and higher reporting of headache. The classical differential clinical manifestations (extra-respiratory symptoms) and analytical data (hyponatraemia and increased levels of AST and creatine kinase) for suspected legionellosis are of questionable value considering their low sensitivity and specificity [4, 26, 27]. However, Mulazimoglu and $\mathrm{YU}_{\mathrm{U}}$ [27] maintain that these data may increase the level of suspicion for legionnaires' disease, particularly for patients who go to the physician late in the course of the disease. The higher frequency of respiratory symptoms, confusion, hyponatraemia and hypoxaemia as well as the BUN increase in sporadic cases may be explained by a higher incidence of underlying pulmonary disease and the greater severity of legionnaires' disease [28]. As observed in other studies, the high frequency of AST elevation in both groups $(48.8 \%$ of the sporadic cases and $31.3 \%$ of the outbreak cases) is noteworthy due to the lower occurrence in community-acquired pneumonia caused by other microorganisms [26, 27].

The mortality of sporadic and epidemic community-acquired legionellosis has decreased in the last decade, due partly to urinary antigen testing, which allows early diagnosis and the detection of milder forms, and partly to the use of more active antibiotics [7, 8, 14, 20, 29]. Sporadic legionnaires' disease was more severe, according to the higher Fine score, and presented the more extensive radiographic abnormalities and the higher frequencies of complications and hospital or ICU admission. These differences may be explained by the higher frequency of severe underlying diseases, the underdiagnosis of sporadic cases, with only the more severely ill patients being detected, and the greater delay in treatment of sporadic cases compared with outbreak-associated cases. The frequency of adequate treatment was similar in the two groups, although the cases of sporadic legionellosis were more often treated with quinolones, which have been reported to produce a faster clinical response than the macrolides [14, 19]. The low mortality observed in the sporadic cases in the present study compared with previous literature may be explained by the early diagnosis and adequate treatment of these cases [7]. The lower mortality of the outbreak cases compared with sporadic cases in the present study (1.8 versus $5.9 \%$ ) agrees with other reports of sporadic legionellosis and community outbreaks [19]. The diagnosis of milder forms of legionnaires' disease and the early treatment in the outbreak setting may justify these differences [14]. The lack of statistically significant differences may be due to the lower number of cases.

The present study has some limitations. The fact that the sporadic and the outbreak cases were studied in two different centres and by two different investigators at different times may represent a bias, especially with regard to risk factors and clinical presentation. However, the investigators followed the same diagnostic protocol, evaluated the same clinical data and used the same definitions of risk factors. Moreover, less severe cases of community-acquired legionellosis not requiring hospital care may have been under-reported in the sporadic case series. Consequently, the characteristics observed in the present study cannot be completely extrapolated to other settings. Different degrees of virulence have been reported among L. pneumophila strains [30], but this aspect was not analysed in the present study.

Several conclusions may be made. First, recognised risk factors for legionellosis are usually necessary for the appearance of the disease, even in an outbreak setting. Secondly, differences found between sporadic and epidemic community-acquired legionnaires' disease regarding risk factors, clinical presentation and outcome may be explained by some characteristics of the cases related to the outbreak. These are: the detection of milder forms of legionellosis, the lower severity of underlying disease and the earlier treatment. Finally, the uncharacteristic presentation of both sporadic and outbreak legionnaires' disease makes it necessary to take this disease into account and thereby perform adequate diagnostic tests. 


\section{REFERENCES}

1 Joseph CA; European Working Group for Legionella Infections, Legionnaires' disease in Europe 2000-2002. Epidemiol Infect 2004; 132: 417-424.

2 Alvarez J, Oyaga N, Escofet A, Codony F, Orcau A, Maria Oliva J. Community-acquired legionellosis in the Barcelona region between 1992 and 1999: epidemiological characteristics and diagnostic methods. Med Clin (Barc) 2001; 117: 495-496.

3 Den Boer JW, Yzerman EP. Diagnosis of Legionella infection in Legionnaires' disease. Eur J Clin Microbiol Infect Dis 2004; 23: 871-878.

4 Gupta SK, Sarosi GA. The role of atypical pathogens in community-acquired pneumonia. Med Clin North Am 2001; 85: 1349-1365.

5 Sopena N, Sabria M, Pedro-Botet ML, et al. Prospective study of community-acquired pneumonia of bacterial etiology in adults. Eur J Clin Microbiol Infect Dis 1999; 18: 852-858.

6 Alvarez J, Dominguez A, Sabria M, et al. Community outbreaks of legionellosis in Catalonia, 1990-2003 [abstract 1324]. In: Abstracts of the $15^{\text {th }}$ European Congress of Clinical Microbiology and Infectious Diseases (Copenhaguen, Denmark). European Society of Clinical Microbiology and Infectious Diseases 2005; 11: Suppl. 2, 424s.

7 Benin AL, Benson RF, Besser RE. Trends in legionnaires' disease, 1980-1998: declining mortality and new patterns of diagnosis. Clin Infect Dis 2002; 35: 1039-1046.

8 Formica N, Yates M, Beers M, et al. The impact of diagnosis by legionella urinary antigen test on the epidemiology and outcomes of Legionnaires' disease. Epidemiol Infect 2001; 127: 275-280.

9 Marston BJ, Lipman HB, Breiman RF. Surveillance for Legionnaires' disease. Risk factors for morbidity and mortality. Arch Intern Med 1994; 154: 2417-2422.

10 Straus WL, Plouffe JF, File TM Jr, et al. Risk factors for domestic acquisition of legionnaires disease. Ohio Legionnaires Disease Group. Arch Intern Med 1996; 156: 1685-1692.

11 Garcia-Fulgueiras A, Navarro C, Fenoll D, et al. Legionnaires' disease outbreak in Murcia, Spain. Emerg Infect Dis 2003; 9: 915-921.

12 Greig JE, Carnie JA, Tallis GF, et al. An outbreak of Legionnaires' disease at the Melbourne Aquarium, April 2000: investigation and case-control studies. Med J Aust 2004; 180: 566-572.

13 Den Boer JW, Yzerman EP, Schellekens J, et al. A large outbreak of Legionnaires' disease at a flower show, the Netherlands, 1999. Emerg Infect Dis 2002; 8: 37-43.

14 Sabria M, Pedro-Botet ML, Gómez J, et al. Fluoroquinolones vs macrolides in the treatment of Legionnaires disease. Chest 2005; 128: 1401-1405.

15 Barrufet-Barque MP, Sauca-Subias G, Force-Sanmartí L, Felip-Benach A, Martinez-Perez E, Capdevila-Morell JA. Legionnaires' disease outbreak. Med Clin (Barc) 2006; 126: 178-182.
16 Fine MJ, Auble TE, Yealy DM, et al. A prediction rule to identify low-risk patients with community-acquired pneumonia. N Engl J Med 1997; 336: 243-250.

17 Edelstein PH, Cianciotto NP. Legionella. In: Mandell GL, Benett JE, Dolin R, eds. Principles and Practice of Infectious Diseases. New York, 16th Edn. Elsevier Churchill Livingstone, 2005; pp. 2711-2724.

18 Lieberman D, Porath A, Schlaeffer F, Lieberman D, Boldur I. Legionella species community-acquired pneumonia. A review of 56 hospitalized adult patients. Chest 1996; 109: 1243-1249.

19 Mykietiuk A, Carratala J, Fernandez-Sabe N, et al. Clinical outcomes for hospitalized patients with Legionella pneumonia in the antigenuria era: the influence of levofloxacin therapy. Clin Infect Dis 2005; 40: 794-799.

20 Fernandez JA, Lopez P, Orozco D, Merino J. Clinical study of an outbreak of Legionnaires' disease in Alcoy, Southeastern Spain. Eur J Clin Microbiol Infect Dis 2002; 21: 729-735.

21 Jerico Alba C, Nogues Solan X, Santos Martinez MJ, et al. Legionella pneumophila pneumonia community epidemic outbreak in Barcelona: "The Barceloneta outbreak". Effect on the early diagnosis and treatment. Rev Clin Esp 2004; 204: 70-74.

22 Monforte R, Estruch R, Vidal J, et al. A community outbreak of Legionnaires' disease in Barcelona: clinical and microbiological study. Med Clin (Barc) 1989; 93: 521525.

23 Sopena N, Pedro-Botet ML, Sabria M, Garcia-Pares D, Reynaga E, Garcia-Nuñez M. Comparative study of community-acquired pneumonia caused by Streptococcus pneumoniae, Legionella pneumophila or Chlamydia pneumoniae. Scand J Infect Dis 2004; 36: 330-334.

24 Watanakunakorn C, Bailey TA. Adult bacteremic pneumococcal pneumonia in a community teaching hospital, 1992-1996. A detailed analyisis of 108 cases. Arch Intern Med 1997; 157: 1965-1971.

25 Pedro-Botet ML, Sabria M, Sopena N, et al. Legionnaires' disease and HIV infection. Chest 2003; 124: 543-547.

26 Sopena N, Sabria-Leal M, Pedro-Botet ML, et al. Comparative study of the clinical presentation of Legionella pneumonia and other community-acquired pneumonias. Chest 1998; 113: 1195-1200.

27 Mulazimoglu L, Yu VL. Can Legionnaires disease be diagnosed by clinical criteria? A critical review. Chest 2001; 120: 1049-1053.

28 Vergis EN, Akbas E, Yu VL. Legionella as a cause of severe pneumonia. Semin Respir Crit Care Med 2000; 21: 295-304.

29 Health $\mathrm{CH}$, Grove DI, Looke DFM. Delaying appropriate therapy of Legionella pneumonia associated with increased mortality. Eur J Clin Microbiol Infect Dis 1996; 15: 286-290.

30 Swanson MS, Hammer BK. Legionella pneumophila pathogenesis: a fateful journey from amoebae to macrophages. Annu Rev Microbiol 2000; 54: 567-613. 